Association between maternal hyperglycemia and composite maternal-birth outcomes

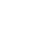

\title{
Running Title: Maternal hyperglycemia and composite maternal-birth outcomes
}

Song-Ying Shen ${ }^{1 \#}$, Li-Fang Zhang ${ }^{1 \#}$, Jian-Rong He ${ }^{1}$, Jin-Hua Lu ${ }^{1}$, Nian-Nian Chen ${ }^{1}$, Wan-Qing Xiao ${ }^{1}$, Ming-Yang Yuan ${ }^{1}$, Hui-Min Xia ${ }^{1}$, Kin Bong Hubert Lam² ${ }^{2}$ Xiu $\mathrm{Qiu}^{1 *}$

${ }^{1}$ Division of Birth Cohort Study, Guangzhou Women and Children's Medical Center, Guangzhou Medical University, Guangzhou, China

${ }^{2}$ Nuffield Department of Population Health, University of Oxford, UK

\# Songying Shen and Lifang Zhang share first authorship.

* Correspondence:

Dr. Xiu Qiu

qxiu0161@163.com, xiu.qiu@bigcs.org;

Dr. Huimin Xia

huimin.xia876001@gmail.com, huimin.xia@bigcs.org 


\section{Abstract}

Objective: The overall impact of maternal hyperglycemia on maternal and birth outcomes is largely underestimated, therefore quantifying the true burden of hyperglycemia in a whole population it is a challenging task. This study aims at examining the association between blood glucose concentration during pregnancy and a composite score of adverse maternal-birth outcomes in a large-scale prospective cohort study in China.

Methods: Pregnant women within "the Born in Guangzhou Cohort Study", China who underwent a standard 75-g oral-glucose-tolerance-test (OGTT) between 22 and 28 gestational weeks were included. A composite score of stillbirth, duration of pregnancy, birth weight, preeclampsia, and cesarean section was developed based on a published maternal-fetal outcomes scale, weighed by the relative severity of the outcomes. Multiple linear regression models were used to assess the associations between OGTT glucose measurements and log composite score. Logistic regression models were used to assess relations with outcome as a categorical variable $(0,1-<3$ and $\geq 3$ ).

Findings: Among 12129 pregnancies, the composite score ranged from 0 to 100 with a median of 2.5 for non-zero values. Elevated fasting glucose level was associated with higher composite score (adjusted coefficients 0.03 [95\% CI, 0.02-0.04] for 1-SD increase). For 1-SD increase in fasting glucose, the risk of having a composite score $1-<3$ and $\geq 3$ rises by $13 \%$ (95\% CI, 8\%-17\%) and $15 \%$ (95\% CI, 7\%-23\%), respectively. Similar association and increase in risk was found for 1-hour and 2-hour glucose.

Conclusion: Elevated fasting, 1-hour, and 2-hour glucose levels are associated with a range of adverse maternal-birth outcomes. The composite score model can be applied to the risk assessment for individual pregnant women and to evaluate the benefits for controlling glucose levels in the population.

Keywords: hyperglycemia, glucose, pregnancy, stillbirth, birth weight, duration of pregnancy, preeclampsia, cesarean section, composite outcome 


\section{INTRODUCTION}

Gestational diabetes mellitus (GDM) is one of the most prevalent major complications during pregnancy worldwide (1-3). Previous studies have linked maternal hyperglycemia to increased risks of various adverse perinatal outcomes, including macrosomia, large for gestational age (LGA), cesarean section, preterm birth, gestational hypertension and hyperbilirubinemia (3, 4). However, all such studies have only investigated the perinatal outcomes in isolation without taking into account the complex inter-relationship between maternal and fetal outcomes, and among the perinatal outcomes themselves; examples include macrosomia and caesarean section (5), gestational hypertension and preterm birth $(6,7)$. In addition, the varying strengths of association with these outcomes, which have different levels of severity and consequences, have not been fully appreciated $(3,4,8)$. This has led to difficulties in assessing the influence of maternal hyperglycemia on both maternal and birth outcomes, and developing optimal management of maternal hyperglycemia, as well as evaluating the effectiveness of glucose-lowering interventions in the general pregnant women population. A comprehensive consideration of multiple adverse outcomes, both in pregnant women and in newborns (9), is therefore needed.

Indeed, some previous studies have attempted to assess multiple perinatal outcomes (8-12). For example, to evaluate the need of care during pregnancy and delivery, Novicoff et al. in 2000 created an outcome score that sums the points of all maternal and fetal outcomes based on relative desirability and frequency of occurrence (10). Verma et al. developed a morbidity assessment index for newborns (called MAIN score), capturing the entire severity spectrum of morbidity at birth to estimate the effectiveness of obstetric interventions on neonatal morbidity $(9,12)$. However, none of these studies have evaluated the influence of maternal hyperglycemia on composite maternal and birth outcomes. A comprehensive measure focusing on capturing GDM-related morbidity in both mother and child should add an important dimension to current understanding of GDM risk and management.

The present study aims to examine the association between blood glucose concentrations during pregnancy, and risk-adjusted adverse maternal-birth outcomes represented by a composite score in a large-scale prospective cohort study in China.

\section{MATERIAL AND METHODS}

\section{Study design and population}

The data used in the present study is part of the Born in Guangzhou Cohort Study (BIGCS), which is a birth cohort study conducted in the Guangzhou Women and Children's Medical Center (GWCMC), China. Study participants were recruited from pregnant women attending their first routine antenatal exam at GWCMC. Inclusion criteria were: residents in Guangzhou, $<20$ weeks gestation, intending to deliver at GWCMC, and planning to remain in Guangzhou with the child for at least 3 years after delivery. A detailed description of the BIGCS protocol has been 
published elsewhere (13). For this analysis, we excluded participants with pre-pregnancy diabetes or chronic hypertension, according to the self-reported medical history. Women with missing blood glucose and delivery data were also excluded (Figure 1).

At enrollment participants underwent an interviewer-administered questionnaire, which collected a wide range of information including socio-demographic data, exposures at home and at workplace, personal lifestyle, medical histories, and health status before pregnancy. The study protocol was approved by GWCMC Ethics Approval Board. Written informed consent was obtained from all participants.

\section{Oral Glucose-Tolerance Test}

Between 22 and 28 weeks of gestation, all pregnant women underwent a standard oral glucose tolerance test (OGTT), during which blood samples $(2 \mathrm{~mL})$ were collected at fasting, 1 hour and 2 hours after a $75 \mathrm{~g}$ glucose load with NaF/EDTA tubes. Detailed procedures of the OGTT test were presented elsewhere (14). Women with GDM diagnosis (based on the International Association of Diabetes and Pregnancy Study Groups [IADPSG] criteria (15) received routine consultation on diet and exercise. They were then asked to self-monitor their preprandial blood glucose levels after having dietary management for 3 to 5 days. Those with fasting blood glucose $\geq 5.3 \mathrm{mmol} / \mathrm{L}$ or $2 \mathrm{~h}$ postprandial glucose $\geq 6.7 \mathrm{mmol} / \mathrm{L}$ after dietary control were prescribed insulin in addition to a diet and exercise regime (16).

\section{Maternal and birth outcomes}

Information about pregnancy complications, mode of delivery, gestational age, birth weight, parity and gender of newborns were obtained from GWCMC's electronic medical records. Gestational age was confirmed by ultrasound examination in the first- or second-trimester. Birth weight was measured by midwives immediately after delivery. Birthweight z scores were calculated using a local population-based birth weight reference (17). LGA was defined as a birthweight larger than the 90th percentile for gestational age by gender and small for gestational age (SGA) was defined as a birthweight lower than the 10th percentile for gestational age by gender, based on the same birth weight reference (17). Preterm birth was defined as birth before 37 weeks of gestation. Spontaneous preterm birth was the birth following spontaneous preterm labor and/or preterm premature rupture of the membranes before 37 weeks of gestation, irrespective of the mode of delivery (vaginal, cesarean section) (18); all this information was obtained from medical records, which was also independently confirmed by two pediatricians. Gestational hypertension was defined as blood pressure $\geq 140 / 90 \mathrm{mmHg}$ on at least two occasions separated by at least 4 hours after 20th week of gestation without the presence of protein in the urine and returned to normal within 12 weeks postpartum (19). Preeclampsia was defined as gestational hypertension combined with proteinuria (protein level in the urine $\geq 300 \mathrm{mg} / 24 \mathrm{~h}$ or $\geq(+)$ by a dipstick test on at least two occasions separated by at least 6 hours)(19). Stillbirth cases were identified from the medical records with the 
10th revision of the International Classification of Diseases (ICD10) codes of O36.401 and P95.

We developed a composite outcome score based on the adverse maternal and infant outcomes related to GDM as suggested by the literature $(3,4)$; maternal-infant outcomes included stillbirth, duration of pregnancy, birth weight, preeclampsia, and cesarean section. A score was assigned to each outcome according to a published unified maternal-fetal outcome risk assessment model, ranging from 0 (perfect outcome) to 100 (maternal or infant death) (10). Each mother-newborn pair was scored based on the existence and severity of the five outcomes as shown in Table 1. A comprehensive outcome score was calculated by summing all above-mentioned outcomes scores for each mother-newborn pair. Higher scores indicated the presence of worse maternal and perinatal outcomes.

\section{Potential confounders}

The following maternal risk factors were selected a priori as potential confounders: age, maternal education level, maternal income, pre-pregnancy body mass index (BMI), maternal smoking status, secondhand smoking (smoke exposure during pregnancy), use of assisted reproduction technology, parity, and gestational age at the time of OGTT. Except for gestational age at OGTT and parity, all information was derived from the self-reported questionnaire at enrollment.

\section{Statistical analysis}

Descriptive statistics of the characteristics and outcomes were reported for the whole sample and across categories of the composite scores. To assess the relationship between OGTT glucose measurements and maternal and fetal outcomes as represented by the composite score, we analyzed the data in two ways. First, the composite score was treated as a continuous variable and was log (base 10) transformed $[\log (\mathrm{score}+1)]$. Multiple linear regression models were used to assess the association between the OGTT glucose measurements and log composite score. Regression coefficients were calculated, representing the change in log composite score for 1-SD change in each fasting, $1 \mathrm{~h}$, and $2 \mathrm{~h}$ glucose measurement. Second, we categorized the composite scores (without transformation) into three groups based on the median of non-zero scores (2.5): 0, low (including scores 1, 2, and 2.5) and high $(\geq 3)$. Logistic regression models were used to evaluate the relationships of the OGTT glucose measurements with composite outcomes score categories, with " 0 " as reference. Odds ratios (ORs) were calculated for a 1-SD increase in each OGTT measurement. The linear and logistic regression models were adjusted for pre-specified potential confounders, including maternal age (continuous), maternal education (middle school or below, college, undergraduate or postgraduate), maternal income ( $<1500,1500-4500,4501-9000, \geq 9001$ Yuan), maternal pre-pregnancy body mass index (BMI, continuous), parity $(0, \geq 1)$, smoking status (yes, no), second-hand smoking exposure during pregnancy (yes, no), use of assisted reproduction technology (yes, no), and gestational age when OGTT was performed 
(continuous). A sensitivity analysis was conducted to include participants who underwent OGTT at 24-28 weeks.

All analyses were performed using SAS 9.3 software (SAS Institute, Cary, NC, USA). P-values $<0.05$ were considered to indicate statistical significance.

\section{RESULTS}

A total of 15,198 pregnant women in BIGCS delivered between February 2012 and January 2016. We excluded women with multiple births, those who dropped out before delivery or terminated their pregnancy, had pre-pregnancy diagnosed hypertension and diabetes, or whose delivery data and OGTT results were missing, resulting in 12,129 mothers and their singleton births in this analysis (Figure 1).

Characteristics of mothers and newborns, and by composite score groups are shown in Table 2. Pregnant women had a mean age of 29.1 years (SD 3.4). The majority of them had attained college or above qualifications and over half of them had a monthly income more than 4500 Yuan. Few women smoked but $30 \%$ were exposed to secondhand smoke during pregnancy. Not unexpectedly, the vast majority $(86.6 \%)$ of the pregnant women were primipara. Only 3.4\% women undertook assisted reproduction technology. About $10 \%$ of the participants were overweight or obese before pregnancy (BMI $\geq 24.0 \mathrm{~kg} / \mathrm{m} 2$, according to the Chinese guidelines (20). The mean plasma glucose levels were $4.3 \mathrm{mmol} / \mathrm{L}$ (SD 0.4) at fasting, $7.7 \mathrm{mmol} / \mathrm{L}$ (SD 1.7) at 1-hour, and $6.6 \mathrm{mmol} / \mathrm{L}$ (SD 1.3) at 2-hour post $75 \mathrm{~g}$ glucose load, respectively. Using the IADPSG criteria, there were 1662 (1662/1662, 100\%) women diagnosed with GDM who received a subsequent diet and exercise advice. Ten $(10 / 1662,0.6 \%)$ women went on to receive insulin therapy. Around $60 \%$ of the pregnancies $(\mathrm{n}=7296)$ resulted in optimal outcome (with a score of 0$)$, one-third $(\mathrm{n}=3935)$ having scores between 1 and $<3$, and $7 \%(\mathrm{n}=898)$ had a composite score $\geq 3$ (20\% being 3 ). There were statistically significant differences in most of the maternal characteristics measured across the three composite score groups.

Overall, the prevalence of preeclampsia $(0.7 \%)$ and stillbirth $(0.1 \%)$ was low, although one-third of deliveries were by cesarean section. Infants were born weighing on average $3191.5 \mathrm{~g}$ (SD 423.9) after a mean gestational length of 38.8 weeks (SD 1.4), with 3.5\% of the babies having spontaneous preterm birth $(<37$ weeks).

Among those who had a non-zero composite score, 4138 (84.2\%) had one outcome, $620(12.6 \%)$ had two, $155(3.2 \%)$ had three or more. Cesarean delivery was the most common outcome, occurring in $4161(85.0 \%)$ of the women with non-zero score.

Table 3 summarizes selected pregnancy-related and birth outcomes by composite score groups. The prevalence of gestational hypertension, preeclampsia, cesarean 
delivery, preterm birth, spontaneous preterm birth, LGA, SGA and stillbirth increased significantly with higher composite score categories.

Table 4 shows the associations between fasting, 1-hour, and 2-hour plasma glucose and composite perinatal outcomes score after adjusted for confounders. Elevated fasting glucose values was significantly associated with higher log composite score as a continuous variable (coefficient 0.03 [95\% CI, 0.02-0.04] for 1-SD increase). For 1-SD increase in fasting glucose, the likelihoods of having composite outcomes score $1-<3$ and $\geq 3$ increased by $13 \%$ (95\% CI, 8\%-17\%) and $15 \%$ (95\% CI, $7 \%-23 \%$ ) in the logistic regression model, respectively. Similar associations were found for 1-hour and 2-hour glucose values. A sensitivity analysis that only included participants who underwent OGTT at 24-28 gestational weeks showed no associations (Table S1).

\section{DISCUSSION}

In the present study, a composite maternal-birth outcome scoring scale based on five perinatal outcomes that are most related to gestational diabetes was adapted from a risk model covering maternal and birth outcomes. Significant associations between increased fasting, 1-hour, and 2-hour post-load plasma glucose levels and increased composite maternal and birth outcomes score were found.

A number of studies have investigated the associations between maternal glucose levels and individual perinatal outcomes, such as preterm birth or $\operatorname{LGA}(3,4)$. However, none has considered the multiple adverse maternal and birth outcomes holistically in the same study. Individual outcomes, e.g. LGA, cesarean delivery, preterm birth, preeclampsia, when considered separately, are unable to capture the overall impact of maternal hyperglycemia in mothers and their newborns, hampering the risk assessment of maternal hyperglycemia and the evaluation of effectiveness and benefit for glucose level management in the general pregnant women population. In the present study, the composite outcome score included birth weight and gestational age, both of which have been widely used as outcome measures of effectiveness of national health policies and interventions for pregnant women, especially in developing countries $(17,18)$, and have been reported to be strongly associated with GDM (via abnormal birthweight and preterm birth) (4, 21). We also included preeclampsia and cesarean section - both associated with elevated glucose concentrations during pregnancy $(4,21)$. Hence, the composite score combining complications in both mother and newborn is an interpretable measure of the actual morbidity that are most related to maternal hyperglycemia during pregnancy.

In the present analysis, we found that the risk of having a higher composite score (between 1 and $<3, \geq 3$ ) decreased for 1-SD lower in fasting, 1-hour and 2-hour glucose levels. This may help to assess the risk of developing adverse maternal and birth outcomes for individual pregnant women and recognize specific needs for 
hyperglycemic women, thus facilitating resource-allocation and care optimization for the high-risk, high-cost patient encounter $(8,9)$. For example, among the women with hyperglycemia, only those who have high risk of overall adverse outcomes need more intensive care and intervention. On the other hand, the linear association between maternal glucose level and the comprehensive outcome score highlights the importance of controlling maternal plasma glucose at low levels, rather than aiming at the level just below the diagnostic cut-off. While there is evidence of substantial benefits from intervention for women with GDM and their newborns, previous intervention studies reported improvement in targeted morbidities separately $(22,23)$ rather than across a spectrum of major morbidities to assess overall health improvement. As a result, clinicians may opt to implement different treatment and management strategies depending on the particular outcome they focus on, which may not necessarily be the best option for the patients. The results also can be applied to evaluate the overall effectiveness and benefit of controlling glucose level in the whole pregnant women population. According to our findings, if the fasting glucose level increases by $1-\mathrm{SD}(0.42 \mathrm{mmol} / \mathrm{L})$ in pregnant women, the risk of having a composite adverse outcomes score $1-<3$ and $\geq 3$ would increase by $13 \%$ and $15 \%$, respectively. Consider China where there is a large number of pregnant women, approximately 20 million per year, an excess of 0.84 million $(13 \% \times 32.4 \%$ [prevalence of women with composite score $1-<3$ ] $\times 20$ million) and 0.22 million $(15 \% \times 7.4 \%$ [prevalence of women with composite score $\geq 3$ ] $\times 20$ million $)$ women per year would suffer from mild and relative severe adverse maternal and birth outcomes if fasting glucose would increase by 1-SD in the population. Adequate control of fasting glucose concentration is therefore necessary in both individual and population levels.

The strengths of our study include the use of high-quality outcome data extracted from medical records and that a wide range of potential confounders have been considered in the analysis. This study had some limitations though. First, we did not adjust for the dietary intake of the women during pregnancy, which could potentially affect fetal growth and other maternal-birth outcomes. Second, we recognize that the weights we applied for the five outcomes in the present study, adapted from the maternal and infant scoring system developed by Novicoff et al in 2000 (10), may not accurately reflect the relative severity of the outcomes. However, there has been no universally agreed standard values and ranking for the outcomes of interest. Once a more appropriate standardized scale for each outcome is developed, studies like ours will be required to confirm the findings. Third, neonatal hypoglycaemia is also an important outcome related to gestational diabetes. Unfortunately, the scale we used did not include hypoglycaemia as an outcome and we also did not collect data about this outcome.

In conclusion, using a composite scale that ranks the relative severity of multiple maternal and infant outcomes, we assessed the effects of elevated fasting, 1-hour, and 2-hour post-load plasma glucose and confirmed the potential benefits of 
315 adequate control of maternal glucose level in improving maternal-birth outcomes as

316 a whole. This could be applied to the risk assessment for individual pregnant women

317 as well as to the evaluation of effectiveness and benefit for controlling glucose level 318 in the population. 


\section{ETHICS STATEMENT}

The study protocol was approved by the Ethics Approval Board of Guangzhou Women and Children's Medical Center. Written informed consent was obtained from all participants.

\section{AUTHOR CONTRIBUTIONS}

XQ and HX designed the study and directed its implementation. SS, JH, JL, NC, WX, and MY were involved in study design, questionnaires development, data collection and follow-up of participants. SS participated in the data analysis and drafted the manuscript. LZ carried out the data analysis, contributed to the writing of the paper. JL managed the data. KL and XQ revised the manuscript. All authors critically revised the manuscript, and approved the final version.

\section{CONFLICT OF INTEREST STATEMENT}

The authors declare that the research was conducted in the absence of any commercial or financial relationships that could be construed as a potential conflict of interest.

\section{ACKNOWLEDGEMENTS}

We thank all the pregnant women who participated in the Born in Guangzhou Cohort Study participants and all staff in the cohort team for their contribution to this study, particularly the research nurses and midwives and other recruiting staff for their excellent work. We thank Professor Ben Willem J. Mol for the contribution of knowledge about the effects of maternal hyperglycemia and Dr. Justina Zurauskiene for help with language editing of the manuscript.

\section{FUNGING}

This study was supported by the Technology and Innovation Commission, Guangzhou, China (201508030037, 201807010086), National Natural Science Foundation of China (81673181, 81803251) and Guangdong Provincial Department of Science and Technology (2014A020213022). 


\section{REFERENCES}

1. Kalter-Leibovici O, Freedman LS, Olmer L, Liebermann N, Heymann A, Tal O, et al. Screening and diagnosis of gestational diabetes mellitus: critical appraisal of the new International Association of Diabetes in Pregnancy Study Group recommendations on a national level. Diabetes Care (2012) 35:1894-6. doi:10.2337/dc12-0041

2. Hirst JE, Tran TS, Do MA, Morris JM, Jeffery HE. Consequences of gestational diabetes in an urban hospital in Viet Nam: a prospective cohort study. PLoS Med (2012) 9:e1001272. doi:10.1371/journal.pmed.1001272

3. Black MH, Sacks DA, Xiang AH, Lawrence JM. Clinical outcomes of pregnancies complicated by mild gestational diabetes mellitus differ by combinations of abnormal oral glucose tolerance test values. Diabetes Care (2010) 33:2524-30. doi:10.2337/dc10-1445

4. Metzger BE, Lowe LP, Dyer AR, Trimble ER, Chaovarindr U, Coustan DR, et al. Hyperglycemia and adverse pregnancy outcomes. N Engl J Med (2008) 358:1991-2002. doi:10.1056/NEJMoa0707943

5. Liu Y, Li G, Chen Y, Wang X, Ruan Y, Zou L, et al. A descriptive analysis of the indications for caesarean section in mainland China. BMC Pregnancy Childbirth (2014) 14:410. doi:10.1186/s12884-014-0410-2

6. Sibai BM. Preeclampsia as a cause of preterm and late preterm (near-term) births. Semin Perinatol (2006) 30:16-9. doi:10.1053/j.semperi.2006.01.008

7. Haedersdal S, Salvig JD, Aabye M, Thorball CW, Ruhwald M, Ladelund S, et al. Inflammatory markers in the second trimester prior to clinical onset of preeclampsia, intrauterine growth restriction, and spontaneous preterm birth. Inflammation (2013) 36:907-13. doi:10.1007/s10753-013-9619-x

8. Roberts CL, Cameron CA, Bell JC, Algert CS, Morris JM. Measuring maternal morbidity in routinely collected health data: development and validation of a maternal morbidity outcome indicator. Med Care (2008) 46:786-94. doi:10.1097/MLR.0b013e318178eae4

9. Verma A, Okun NB, Maguire TO, Mitchell BF. Morbidity assessment index for newborns: a composite tool for measuring newborn health. Am J Obstet Gynecol (1999) 181:701-8.

10. Novicoff WM, Wagner DP, Knaus WA, Kane EK, Cecere F, Draper E, et al. Initial development of a system-wide maternal-fetal outcomes assessment program. Am J Obstet Gynecol (2000) 183:291-300. doi:10.1067/mob.2000.108087

11. Oken E, Kleinman KP, Belfort MB, Hammitt JK, Gillman MW. Associations of gestational weight gain with short- and longer-term maternal and child health outcomes. Am J Epidemiol (2009) 170:173-80. doi:10.1093/aje/kwp101

12. Verma A, Weir A, Drummond J, Mitchell BF. Performance profile of an outcome measure: morbidity assessment index for newborns. J Epidemiol Community Health (2005) 59:420-6. doi:10.1136/jech.2003.019109

13. Qiu X, Lu JH, He JR, Lam KH, Shen SY, Guo Y, et al. The Born in Guangzhou Cohort Study (BIGCS). Eur J Epidemiol (2017) 32:337-46. 
doi:10.1007/s10654-017-0239-x

14. Shen S, Lu J, Zhang L, He J, Li W, Chen N, et al. Single Fasting Plasma Glucose Versus 75-g Oral Glucose-Tolerance Test in Prediction of Adverse Perinatal Outcomes: A Cohort Study. EBioMedicine (2017) 16:284-91. doi:10.1016/j.ebiom.2017.01.025

15. Metzger BE, Gabbe SG, Persson B, Buchanan TA, Catalano PA, Damm P, et al. International association of diabetes and pregnancy study groups recommendations on the diagnosis and classification of hyperglycemia in pregnancy. Diabetes Care (2010) 33:676-82. doi:10.2337/dc09-1848

16. Obstetrics Subgroup, Chinese Society of Obstetrics and Gynecology, Chinese Medical Association, Group of Pregnancy with Diabetes Mellitus, Chinese Society of Perinatal Medicine, Chinese Medical Association. [Diagnosis and therapy guideline of pregnancy with diabetes mellitus]. Zhonghua Fu Chan Ke Za Zhi (2014) 49:561-9.

17. He JR, Xia HM, Liu Y, Xia XY, Mo WJ, Wang P, et al. A new birthweight reference in Guangzhou, southern China, and its comparison with the global reference. Arch Dis Child (2014) 99:1091-7. doi:10.1136/archdischild-2013-305923

18. Goldenberg RL, Culhane JF, Iams JD, Romero R. Epidemiology and causes of preterm birth. Lancet (2008) 371:75-84. doi:10.1016/S0140-6736(08)60074-4

19. Ye C, Ruan Y, Zou L, Li G, Li C, Chen Y, et al. The 2011 survey on hypertensive disorders of pregnancy (HDP) in China: prevalence, risk factors, complications, pregnancy and perinatal outcomes. PLoS One (2014) 9:e100180. doi:10.1371/journal.pone.0100180

20. Chen C, Lu FC. The guidelines for prevention and control of overweight and obesity in Chinese adults. Biomed Environ Sci (2004) 17 Suppl:1-36

21. Langer O, Yogev Y, Most O, Xenakis EM. Gestational diabetes: the consequences of not treating. Am J Obstet Gynecol (2005) 192:989-97. doi:10.1016/j.ajog.2004.11.039

22. Landon MB, Spong CY, Thom E, Carpenter MW, Ramin SM, Casey B, et al. A multicenter, randomized trial of treatment for mild gestational diabetes. $N$ Engl J Med (2009) 361:1339-48. doi:10.1056/NEJMoa0902430

23. Crowther CA, Hiller JE, Moss JR, McPhee AJ, Jeffries WS, Robinson JS. Effect of treatment of gestational diabetes mellitus on pregnancy outcomes. $N$ Engl J Med (2005) 352:2477-86. doi:10.1056/NEJMoa042973 
$430 \quad$ Figure legends

431 FIGURE 1| The flowchart of the study. 
432 TABLE $1 \mid$ Maternal-birth outcomes scoring scale.

\begin{tabular}{ll}
\hline Outcomes & Score* \\
\hline Stillbirth & 100 \\
Preeclampsia & 5 \\
Cesarean delivery & 2.5 \\
Gestational age $\leq 24 \mathrm{wk}$ & 11 \\
Gestational age $25-26 \mathrm{wk}$ & 7 \\
Gestational age $27-28 \mathrm{wk}$ & 6 \\
Gestational age $29-30 \mathrm{wk}$ & 4 \\
Gestational age $31-33 \mathrm{wk}$ & 3 \\
Gestational age $34-36 \mathrm{wk}$ & 2 \\
Gestational age $\geq 37 \mathrm{wk}$ and $<42 \mathrm{wk}$ & 0 \\
Gestational age $>42 \mathrm{wk}$ & 1 \\
Birth weight $<750 \mathrm{~g}$ & 10 \\
Birth weight $750-1000 \mathrm{~g}$ & 7 \\
Birth weight $1000-1500 \mathrm{~g}$ & 5 \\
Birth weight $1500-2500 \mathrm{~g}$ & 3 \\
Birth weight $2500-4000 \mathrm{~g}$ & 0 \\
Birth weight $>4000 \mathrm{~g}$ & 1 \\
\hline
\end{tabular}

$433 *$ The score assigned to each outcome was referred to a published unified maternal-fetal outcome risk

434 assessment model, ranging from 0 (perfect outcome) to 100 (maternal or infant death) (10). 
TABLE 2| Characteristics of the 12129 study participants and their newborns by different groups of composite score.

\begin{tabular}{|c|c|c|c|c|c|}
\hline \multirow{2}{*}{ Characteristic } & \multirow{2}{*}{$\begin{array}{c}\text { All } \\
\text { participants }\end{array}$} & \multicolumn{4}{|c|}{ Composite score } \\
\hline & & $\mathbf{0}$ & $1-<3$ & $\geq \mathbf{3}$ & $p$-value \\
\hline $\mathrm{n}$ & 12129 & 7296 & 3935 & 898 & \\
\hline Age (years), mean $\pm \mathrm{SD}$ & $29.1 \pm 3.4$ & $28.6 \pm 3.1$ & $29.9 \pm 3.7$ & $29.5 \pm 3.6$ & $<0.0001$ \\
\hline \multicolumn{6}{|l|}{$\begin{array}{l}\text { Plasma glucose }(\mathrm{mmol} / \mathrm{L}) \\
\text { mean } \pm \mathrm{SD}\end{array}$} \\
\hline Fasting & $4.3 \pm 0.4$ & $4.3 \pm 0.4$ & $4.3 \pm 0.4$ & $4.3 \pm 0.5$ & $<0.0001$ \\
\hline 1-hour & $7.7 \pm 1.7$ & $7.6 \pm 1.6$ & $7.8 \pm 1.7$ & $7.9 \pm 1.8$ & $<0.0001$ \\
\hline 2-hour & $6.6 \pm 1.3$ & $6.5 \pm 1.3$ & $6.8 \pm 1.4$ & $6.8 \pm 1.5$ & $<0.0001$ \\
\hline $\begin{array}{l}\text { Length of gestation at time of } \\
\text { OGTT (wk), mean } \pm \text { SD }\end{array}$ & $24.5 \pm 1.6$ & $24.5 \pm 1.6$ & $24.5 \pm 1.6$ & $24.4 \pm 1.6$ & 0.441 \\
\hline Income (Yuan), n (\%) & & & & & $<0.0001$ \\
\hline$<1500$ & $1115(9.6)$ & $674(9.7)$ & $363(9.7)$ & $78(9.3)$ & \\
\hline $1500-4500$ & 3460 (29.9) & 2207 (31.6) & $1001(26.7)$ & $255(30.4)$ & \\
\hline $4501-9000$ & $4947(42.8)$ & $2933(42.0)$ & $1641(43.8)$ & $373(44.5)$ & \\
\hline$\geq 9001$ & $2040(17.7)$ & $1166(16.7)$ & $741(19.8)$ & $133(15.9)$ & \\
\hline Education, n (\%) & & & & & 0.0051 \\
\hline Middle school or below & $1112(9.2)$ & $645(8.8)$ & $377(9.6)$ & $90(10.0)$ & \\
\hline College & $3068(25.3)$ & $1847(25.3)$ & 969 (24.6) & $252(28.1)$ & \\
\hline Undergraduate & $6472(53.4)$ & 3929 (53.9) & $2067(52.5)$ & $476(53.0)$ & \\
\hline Postgraduate & $1471(12.2)$ & $875(12.0)$ & $522(13.3)$ & $80(8.9)$ & \\
\hline $\begin{array}{l}\text { Pre-pregnancy body mass } \\
\text { index }\left(\mathrm{kg} / \mathrm{m}^{2}\right) \text {, mean } \pm \mathrm{SD} \text { and } \\
\mathrm{n}(\%)\end{array}$ & $20.4 \pm 2.7$ & $20.1 \pm 2.5$ & $20.8 \pm 2.8$ & $20.7 \pm 3.0$ & $<0.0001$ \\
\hline$\leq 18.5$ & $2967(25.0)$ & 1994 (27.9) & $768(20.0)$ & 205 (23.6) & $<0.0001$ \\
\hline $18.5-23.9$ & 7785 (65.6) & $4634(64.8)$ & $2598(67.5)$ & $553(63.6)$ & \\
\hline $24.0-27.9$ & 939 (7.9) & $450(6.3)$ & $398(10.3)$ & $91(10.5)$ & \\
\hline$\geq 28.0$ & $181(1.5)$ & $74(1.0)$ & $86(2.2)$ & $21(2.4)$ & \\
\hline $\begin{array}{l}\text { Smoking during pregnancy, } \mathrm{n} \\
(\%)\end{array}$ & $59(0.5)$ & $34(0.5)$ & $16(0.4)$ & $9(1.0)$ & 0.0584 \\
\hline $\begin{array}{l}\text { Passive smoking during } \\
\text { pregnancy, } \mathrm{n}(\%)\end{array}$ & 3494 (29.6) & $2166(30.4)$ & $1082(28.3)$ & $246(28.5)$ & 0.0467 \\
\hline Parity at enrollment $\geq 1, \mathrm{n}(\%)$ & $1613(13.4)$ & $845(11.7)$ & $654(16.8)$ & $114(12.8)$ & $<0.001$ \\
\hline $\begin{array}{l}\text { Use of assisted reproduction } \\
\text { technology, } \mathrm{n}(\%)\end{array}$ & $396(3.4)$ & $182(2.6)$ & $168(4.4)$ & $46(5.3)$ & $<0.0001$ \\
\hline
\end{tabular}


TABLE 3| Obstetrical and newborn outcomes of the 12129 study participants.

\begin{tabular}{|c|c|c|c|c|}
\hline \multirow[b]{2}{*}{ Characteristic or outcome } & \multirow[b]{2}{*}{ All participants } & \multicolumn{3}{|c|}{ Composite score } \\
\hline & & $\mathbf{0}$ & $1-<3$ & $\geq \mathbf{3}$ \\
\hline $\mathbf{n}$ & 12129 & 7296 & 3935 & 898 \\
\hline \multicolumn{5}{|l|}{ Obstetrical outcomes } \\
\hline Hypertension, n (\%) & $295(2.4)$ & $82(1.1)$ & $100(2.5)$ & $113(12.8)$ \\
\hline Gestational hypertension & $214(1.8)$ & $82(1.1)$ & $100(2.5)$ & $32(3.6)$ \\
\hline Preeclampsia & $81(0.7)$ & - & - & $81(9.15)$ \\
\hline Cesarean delivery, n (\%) & $4161(34.3)$ & - & $3639(92.5)$ & $522(59.0)$ \\
\hline \multicolumn{5}{|l|}{ Newborn outcomes } \\
\hline Stillbirth, n (\%) & $13(0.1)$ & - & - & $13(1.4)$ \\
\hline $\begin{array}{l}\text { Gestational age at delivery } \\
\text { (week), mean } \pm \text { SD }\end{array}$ & $38.8 \pm 1.4$ & $39.0 \pm 1.0$ & $38.9 \pm 1.2$ & $36.7 \pm 2.7$ \\
\hline Preterm birth, n (\%) & $579(4.8)$ & - & $188(4.8)$ & $391(44.5)$ \\
\hline $\begin{array}{l}\text { Spontaneous preterm birth, } \mathrm{n} \\
(\%)\end{array}$ & $419(3.5)$ & - & $169(4.3)$ & $250(33.9)$ \\
\hline Birth weight $(\mathrm{g})$, mean \pm SD & $3191.5 \pm 423.9$ & $3194.5 \pm 316.8$ & $3292.0 \pm 373.8$ & $2720.5 \pm 863.5$ \\
\hline $\begin{array}{l}\text { Birth weight Z-score, mean } \pm \\
\text { SD }\end{array}$ & $0.1 \pm 1.0$ & $0.0 \pm 0.8$ & $0.3 \pm 0.9$ & $-0.2 \pm 1.8$ \\
\hline \multicolumn{5}{|l|}{$\begin{array}{l}\text { Birth weight for gestational } \\
\text { age, n }(\%)\end{array}$} \\
\hline SGA & $875(7.3)$ & $431(5.9)$ & $122(3.1)$ & $322(36.7)$ \\
\hline AGA & $9917(82.3)$ & $6354(87.6)$ & $3210(81.9)$ & $353(40.2)$ \\
\hline LGA & $1254(10.4)$ & $465(6.4)$ & $586(15.0)$ & $203(23.1)$ \\
\hline
\end{tabular}

439 Abbreviations: SGA, small for gestational age, AGA, appropriate for gestational age, LGA, large for 440 gestational age. 
441 TABLE 4| Adjusted Coefficients and Odds Ratios for Associations between Maternal

442 Glycemia and Composite Maternal-birth Outcomes.

\begin{tabular}{|c|c|c|c|c|c|c|c|c|}
\hline \multirow{3}{*}{ Composite score } & & \multicolumn{7}{|c|}{ Plasma Glucose Level } \\
\hline & & \multirow{2}{*}{$\mathbf{n}$} & \multicolumn{2}{|c|}{ Fasting } & \multicolumn{2}{|c|}{ At 1-hour } & \multicolumn{2}{|c|}{ At 2-hour } \\
\hline & & & Crude & Adjusted $^{\text {a }}$ & Crude & Adjusted a & Crude & Adjusted $^{\text {a }}$ \\
\hline \multirow{2}{*}{\multicolumn{2}{|c|}{$\begin{array}{l}\text { Continuous, coefficients } \\
(95 \% \mathrm{CI})^{\mathrm{b}} \\
\text { Categorical, OR }(95 \% \mathrm{CI})\end{array}$}} & 12129 & $0.05(0.04,0.07)$ & $0.03(0.02,0.04)$ & $0.05(0.04,0.06)$ & $0.02(0.01,0.04)$ & $0.06(0.05,0.07)$ & $0.03(0.02,0.04)$ \\
\hline & & & & & & & & \\
\hline \multicolumn{2}{|l|}{0} & 7296 & Ref. & Ref. & Ref. & Ref. & Ref. & Ref. \\
\hline \multicolumn{2}{|l|}{$1-<3$} & 3935 & $1.20(1.15,1.25)$ & $1.13(1.08,1.17)$ & $1.16(1.12,1.21)$ & $1.06(1.02,1.11)$ & $1.19(1.15,1.24)$ & $1.09(1.05,1.14)$ \\
\hline \multicolumn{2}{|l|}{$\geq 3$} & 898 & $1.21(1.13,1.29)$ & $1.15(1.07,1.23)$ & $1.22(1.14,1.30)$ & $1.15(1.07,1.24)$ & $1.22(1.14,1.30)$ & $1.14(1.06,1.22)$ \\
\hline 443 & \multicolumn{8}{|c|}{ Abbreviations: CI, confidence interval, OR, odd ratio. } \\
\hline 444 & \multicolumn{8}{|c|}{${ }^{\text {a }}$ Regression coefficients or odds ratios were for an increase in the glucose level of $1 \mathrm{SD}(0.42 \mathrm{mmol} / \mathrm{L}$ for the } \\
\hline 445 & \multicolumn{8}{|c|}{ fasting glucose level, $1.66 \mathrm{mmol} / \mathrm{L}$ for the $1 \mathrm{~h}$ glucose level, and $1.35 \mathrm{mmol} / \mathrm{L}$ for the $2 \mathrm{~h}$ glucose level.). Adjusted } \\
\hline 446 & \multicolumn{8}{|c|}{ for age, income, educational level, smoking during pregnancy, second hand smoking exposure during pregnancy, } \\
\hline 447 & \multicolumn{8}{|c|}{ pre-pregnancy BMI, parity, assisted reproductive technology, gestational age at the OGTT. } \\
\hline 448 & \multicolumn{8}{|c|}{${ }^{\mathrm{b}}$ Composite score was $\log$ transformed as $\log ($ score +1$)$. } \\
\hline \multicolumn{9}{|l|}{449} \\
\hline \multicolumn{9}{|l|}{450} \\
\hline
\end{tabular}

